\title{
OPEN A neutralizing IL-11 antibody reduces vessel hyperplasia in a mouse carotid artery wire injury model
}

David Schumacher ${ }^{1,2,15}$, Elisa A. Liehn ${ }^{3,4,5,6,15}$, Pakhwan Nilcham², David Castaño Mayan 7,8,9, Chutima Rattanasopa ${ }^{7}$, Kaviya Anand ${ }^{7}$, Gustavo E. Crespo-Avilan ${ }^{6,10,11}$, Sauri Hernandez-Resendiz ${ }^{6,10}$, Roshni R. Singaraja ${ }^{7,8,9}$, Stuart A. Cook ${ }^{6,10,12,16}$ \& Derek J. Hausenloy $6,8,10,13,14,16 \bowtie$

Vascular restenosis remains a major problem in patients with coronary artery disease (CAD) and peripheral artery disease (PAD). Neointimal hyperplasia, defined by post-procedure proliferation and migration of vascular smooth muscle cells (VSMCs) is a key underlying pathology. Here we investigated the role of Interleukin 11 (IL-11) in a mouse model of injury-related plaque development. Apoe $^{-l-}$ mice were fed a hyperlipidaemic diet and subjected to carotid wire injury of the right carotid. Mice were injected with an anti-IL11 antibody (X203), IgG control antibody or buffer. We performed ultrasound analysis to assess vessel wall thickness and blood velocity. Using histology and immunofluorescence approaches, we determined the effects of IL-11 inhibition on VSMC and macrophages phenotypes and fibrosis. Treatment of mice with carotid wire injury using X203 significantly reduced post-endothelial injury vessel wall thickness, and injury-related plaque, when compared to control. Immunofluorescence staining of the injury-related plaque showed that X203 treatment did not reduce macrophage numbers, but reduced the number of VSMCs and lowered matrix metalloproteinase 2 (MMP2) levels and collagen content in comparison to control. X203 treatment was associated with a significant increase in smooth muscle protein $22 \alpha$ (SM22 $\alpha$ ) positive cells in injury-related plaque compared to control, suggesting preservation of the contractile VSMC phenotype. Interestingly, X203 also reduced the collagen content of uninjured carotid arteries as compared to IgG, showing an additional effect on hyperlipidemia-induced arterial remodeling in the absence of mechanical injury. Therapeutic inhibition of IL-11 reduced vessel wall thickness, attenuated neointimal hyperplasia, and has favorable effects on vascular remodeling following wire-induced endothelial injury. This suggests IL-11 inhibition as a potential novel therapeutic approach to reduce arterial stenosis following revascularization in CAD and PAD patients.

\footnotetext{
${ }^{1}$ Institute of Experimental Medicine and Systems Biology, University Hospital, RWTH Aachen University, Aachen, Germany. '2Department of Anesthesiology, University Hospital, RWTH Aachen University, Aachen, Germany. ${ }^{3}$ Department of Cardiology, Angiology and Intensive Medicine, University Hospital Aachen, Aachen, Germany. ${ }^{4}$ Victor Babes National Institute of Pathology, Bucharest, Romania. ${ }^{5}$ Department of Intensive Care and Intermediate Care, University Hospital, RWTH Aachen University, Aachen, Germany. ${ }^{6}$ National Heart Research Institute Singapore, National Heart Centre, Singapore 169609, Singapore. ${ }^{7}$ Translational Laboratories in Genetic Medicine, Agency for Science, Research and Technology, Singapore 138648, Singapore. ${ }^{8}$ Yong Loo Lin School of Medicine, National University Singapore, Singapore 169857, Singapore. ${ }^{9}$ Cardiovascular Research Institute, National University Health System, Singapore 119228, Singapore. ${ }^{10}$ Cardiovascular and Metabolic Disorders Program, Duke-National University of Singapore Medical School, 8 College Road, Singapore 169857, Singapore. ${ }^{11}$ Department of Biochemistry, Medical Faculty, Justus Liebig-University, Giessen, Germany. ${ }^{12} \mathrm{MRC}$ LMS, London W12 0NN, UK. ${ }^{13}$ The Hatter Cardiovascular Institute, University College London, London WC1E 6BT, UK. ${ }^{14}$ Cardiovascular Research Center, College of Medical and Health Sciences, Asia University, Taichung, Taiwan. ${ }^{15}$ These authors contributed equally: David Schumacher and Elisa A. Liehn. ${ }^{16}$ These authors jointly supervised this work: Stuart A. Cook and Derek J. Hausenloy. ${ }^{\circledR}$ email: derek.hausenloy@duke-nus.edu.sg
} 


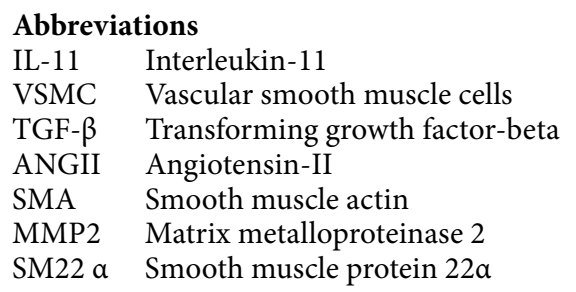

Despite advances in stent design and revascularization therapies, vascular restenosis remains a major problem in patients with coronary artery disease $(\mathrm{CAD})$ and peripheral artery disease $(\mathrm{PAD})^{1-4}$. In-stent restenosis can lead to severe complications such as cardiac ischemia and chronic limb threatening ischemia, and new therapeutic strategies are needed to prevent these complications. Vascular smooth muscle cells (VSMCs) switching from its contractile phenotype to a synthetic phenotype is a major contributor to neointimal hyperplasia, the key pathology underlying vascular restenosis ${ }^{2,3}$.

VSMCs are specialized cells found within the medial layer of the vasculature where their primary function is to regulate vessel tone and blood pressure. In response to vascular injury, VSMCs proliferate, migrate into the tunica intima and assume a synthetic phenotype which is an adaptive response but results in vessel wall thickening. The synthetic VSMC phenotype is characterized by secretion of extracellular matrix, leading to fibrosis and inflammation. The cellular transition to a synthetic phenotype is termed phenotypic switching and plays a key role in arterial restenosis, aortic remodelling, and the development of atherosclerosis ${ }^{5-11}$.

Two key factors associated with VSMC phenotypic switching and vascular pathologies such as atherosclerosis and arterial restenosis are transforming growth factor-beta (TGF $\beta$ ) and angiotensin-II (ANGII) ${ }^{12-14}$. Fibroblastto-myofibroblast differentiation and VSMC phenotypic switching share many similarities, including the secretion of extracellular matrix, cell proliferation and migration, and both transitions can be triggered by the same stimuli.

We have recently discovered that IL-11, a little studied cytokine of the IL-6 family, is important for fibroblast activation downstream of both TGF $\beta 1$ and ANGII as well as for VSMC phenotypic switching, in response to the same stimuli ${ }^{15,16}$. We hypothesized that IL-11 might play a role in vessel hyperplasia and investigated the effects of the neutralizing IL-11 antibody (X203) or an isotype control IgG antibody in a carotid wire-induced endothelial injury mouse model.

\section{Material and methods}

All experiments and methods were performed in accordance with relevant guidelines and regulations. All animal experiments were performed in accordance with ARRIVE (Animal Research: Reporting of In Vivo Experiments) guidelines and approved by the Biomedical Sciences Institute Singapore Institutional Animal Care Committee at $A^{\star S T A R}$ (161165). All methods are reported in accordance with ARRIVE guidelines.

Mouse husbandry. All experiments were approved by the Biomedical Sciences Institute Singapore Institutional Animal Care Committee at $A^{\star}$ STAR (161165). Mice were maintained on a $12 \mathrm{~h}$ dark-light cycle, with ad libitum access to water and were fed with lipid-rich Western-Type Diet (D12079B, Research Diets, NJ), as indicated. Plasma for all experiments was isolated from blood withdrawn from the orbital sinus in EDTA coated capillary tubes. Plasma alanine aminotransferase (ALT), aspartate aminotransferase (AST), low-density lipoprotein (LDL-C), high-density lipoprotein (HDL-C), triglycerides (TG) and total cholesterol were measured using Cobas c111 (Roche Diagnostics, Switzerland).

Carotid artery wire injury model of vascular restenosis. Male, 10 to 12 week Apoe $e^{-/-}$mice (C57BL/6J background, Charles River Laboratory, Italy) were fed lipid-rich Western-Type Diet ${ }^{17}$ for a total of 3 weeks: 1 week before and 2 weeks after wire injury. Only male mice were used for this study to avoid the interference of estrogen effects on injury-related plaque with our target of interest IL-11. For the wire injury procedure, mice were anesthetized $(100 \mathrm{mg} / \mathrm{kg}$ ketamine hydrochloride, $10 \mathrm{mg} / \mathrm{kg}$ xylazine i.p.) and subjected to endothelial denudation of the left common carotid artery using a $1 \mathrm{~cm}$ insertion of a flexible $0.36 \mathrm{~mm}$ guide wire through a transverse arteriotomy of the external carotid artery, as previously described ${ }^{17}$. Prior to surgery and for up to 2 days post-surgery, we performed analgesia with subcutaneous injection of Buprenorphine $(0.05-0.1 \mathrm{mg} / \mathrm{kg})$. Mice were randomly divided in three treatment groups: (1) PBS, (2) IgG, and (3) anti-IL-11 antibody treatment. Twenty mg/kg anti-IL-11 mouse monoclonal antibody (Clone 3C6; X203) or control IgG (Clone 11E10) were administered via intra-peritoneal injections ${ }^{16}$, twice per week beginning on the day of wire injury and continuing for 2 weeks (Fig. 1A).

X203 was generated in mice using a cDNA encoding amino acid 22-199 of human IL-11 cloned into expression plasmids (Aldevron Freiburg GmbH, Freiburg, Germany), as described previously ${ }^{18}$. Its efficacy has already been demonstrated in arterial remodeling ${ }^{16}$, myocardial infarction ${ }^{15}$, liver fibrosis ${ }^{19}$ and pulmonary fibrosis ${ }^{18}$.

Ultrasound measurements of the carotid arteries and heart. Mice were anesthetized with $2 \%$ isoflurane and monitored to maintain heart rate above 500 beats/min during measurements. Measurements were performed in B-Mode and M-Mode. Velocities were recorded and measured in B-Mode (2D-realtime) using angle correction and vessel diameters (wall thickness) were recorded and analyzed in M-Mode using a $40 \mathrm{MHz}$ transducer and a small-animal ultrasound imager (Vevo 3100, FUJIFILM Visualsonics, Toronto, Canada) as well as the VevoLab Software (FUJIFILM Visualsonics, Toronto, Canada). 
A

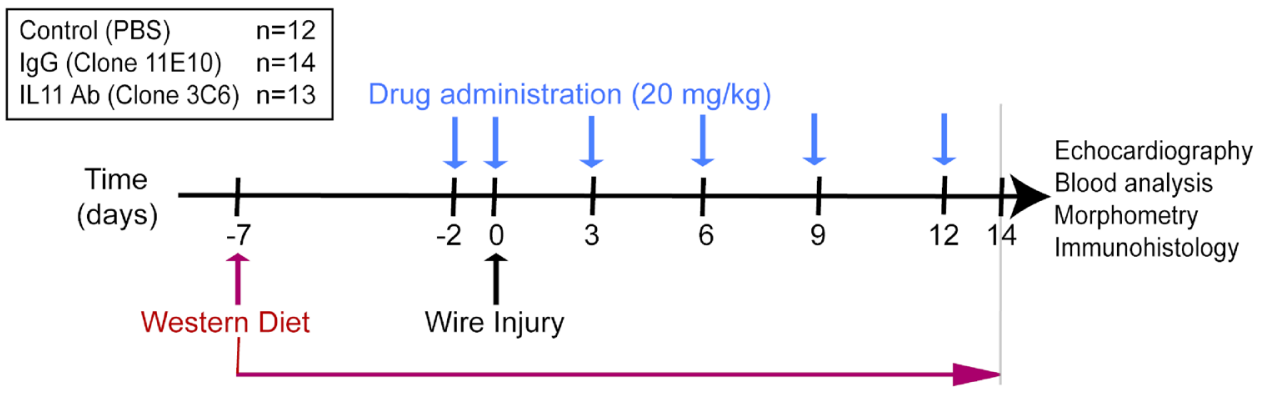

B
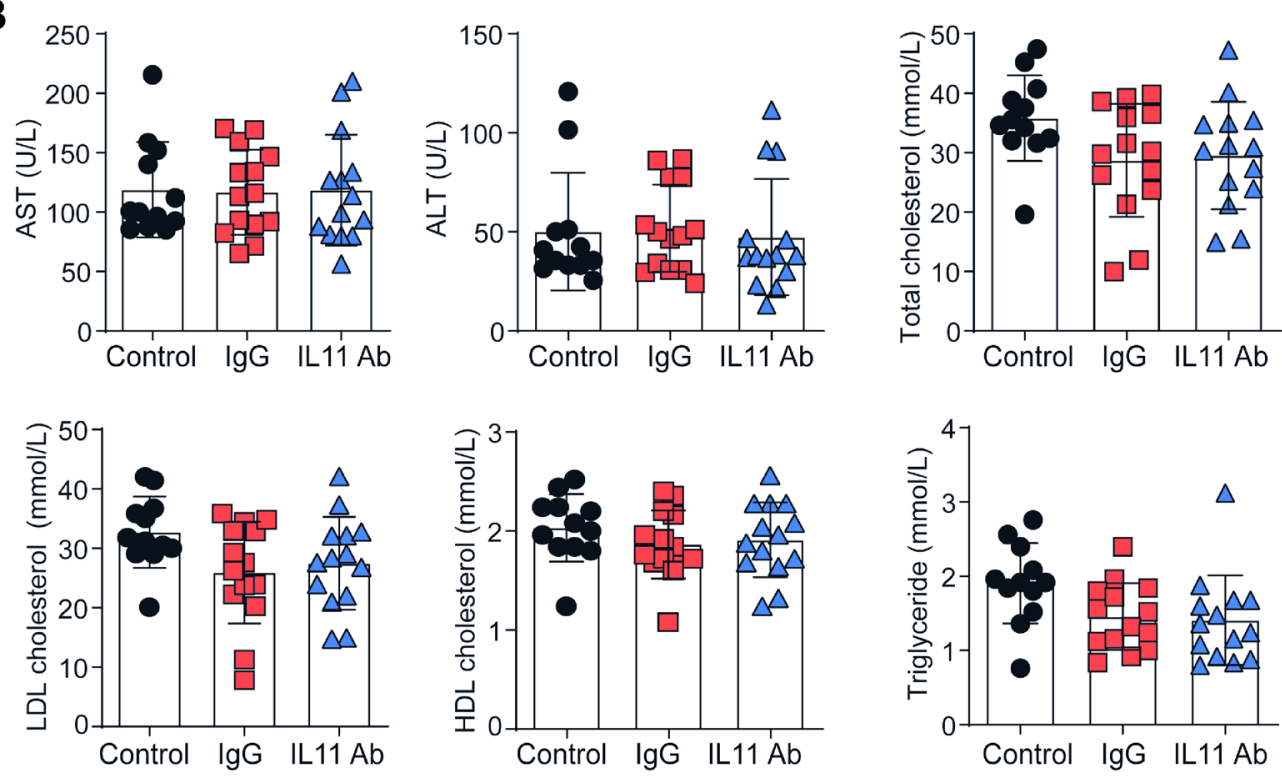

Figure 1. The effect of anti-IL-11 antibody (X203) treatment on plasma lipids. (A) A schematic of the experimental design. (B) Plasma concentration of AST, ALT, triglycerides, total cholesterol, LDL cholesterol and HDL cholesterol ( $\mathrm{N}=12-14$ /group, One-way ANOVA, Tukey's multiple comparison test, Values: mean $\pm \mathrm{SD}$ ).

Histology and immunohistochemistry. Two weeks following wire injury, mice were anesthetized (100 mg/kg ketamine, $10 \mathrm{mg} / \mathrm{kg}$ xylazine, i.p.) and carotid arteries were excised, fixed in formalin and embedded in paraffin. The carotid arteries were then cut in $5 \mu \mathrm{m}$ serial sections starting from the bifurcation until $500 \mu \mathrm{m}$, for all the collected sections. Verhoeff Van Gieson Elastic stain was performed as recommended by the manufacturer (ab150667, Abcam, Cambridge, UK) in 10 serial sections (50 $\mu \mathrm{m}$ apart, starting from the bifurcation) for the left side and 4 serial sections ( $50 \mu \mathrm{m}$ apart, starting from the bifurcation for the right side). Injuryrelated plaque areas were determined for all sections using Diskus software (Hilgers, Königswinter, Germany), as previously described ${ }^{17}$. The average of all 10 sections for the left side ( 4 for right side) was considered as final restenosis area for each vessel.

For further measurements and to minimize variability of arterial layers after mechanical injury, we performed the measurements in whole vessel wall and have referred to it as injury-related plaque. Serial sections ( 3 sections per mouse, $100 \mu \mathrm{m}$ apart) were stained to analyze the injury-related plaque and vessel for early differentiation of VSMCs (SM22 $\alpha$ ab14106, Abcam, Cambridge, UK) and mature VSMCs (smooth muscle actin, M 0851 clone 1A4, DAKO, Germany), macrophages (Mac2, CL8942AP, Cedarlane, Germany) and MMP2 (ab110186, Abcam, Cambridge, UK). The sections were counterstained with DAPI for quantification of total cells. Positive-stained cells were counted in the injury-related plaque in each section and expressed as cells per injury-related plaque, percentage of all cells or percentage of positive area from the total injury-related plaque area. The results are represented as average of the measurements of all 3 slides.

Three serial sections, $100 \mu \mathrm{m}$ apart, were stained with Gomori's 1-step trichrome stain (ab150686, Abcam, Cambridge, UK). Blue-stained collagen content was analyzed with Cell P Software (Olympus, Hamburg, Germany) and expressed as a percentage of the injury-related plaque area. Final results were represented as average of the measurements of all 3 slides. 
A

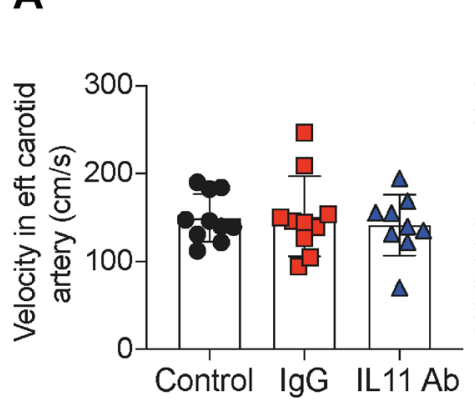

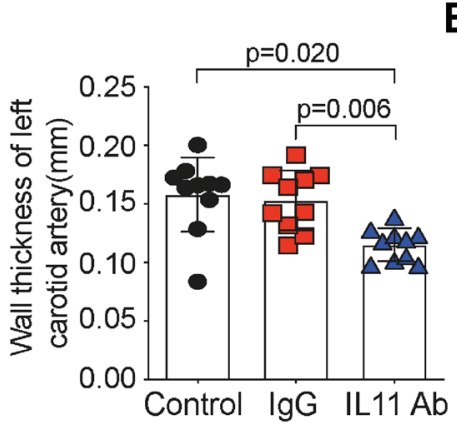

B

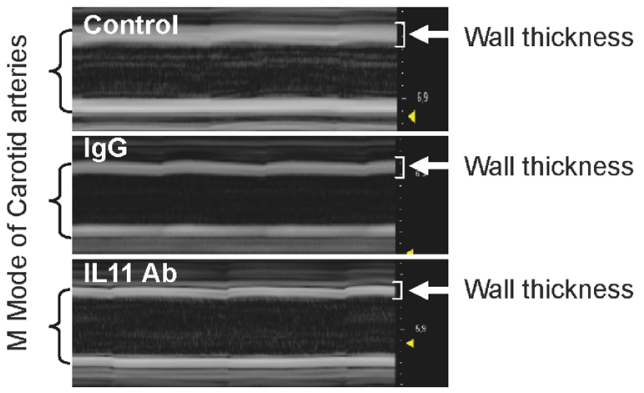

C

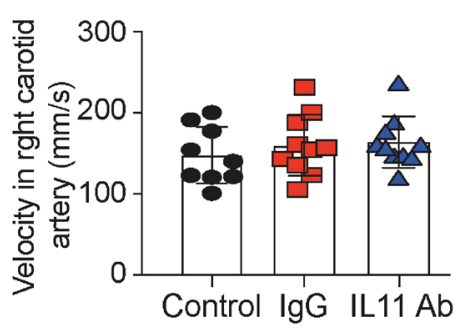

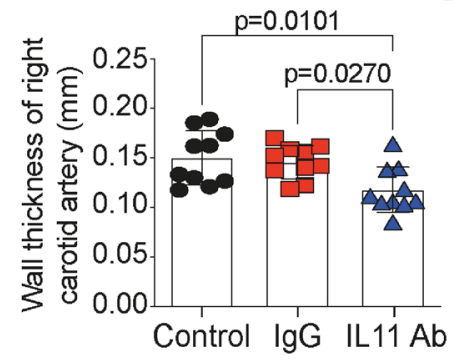

D

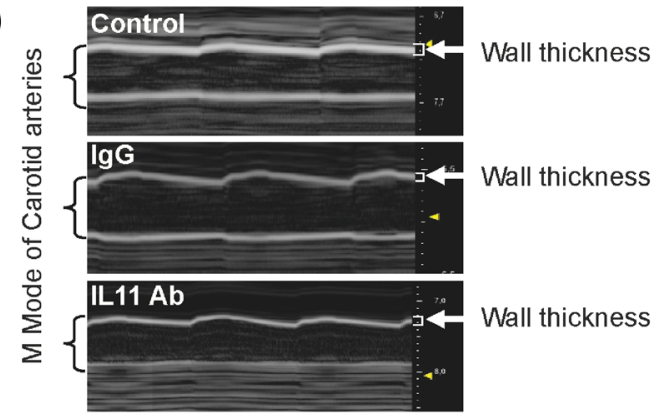

Figure 2. The effect of anti-IL-11 antibody (X203) treatment on vessel wall thickness. (A) Velocity and wall thickness of injured left carotid artery ( $\mathrm{N}=10$ /group, One-way ANOVA, Tukey's multiple comparison test, Values \pm SD). (B) Representative M-Mode images of injured left carotid artery. Brackets show carotid artery size, and arrows point out the measured wall thickness. (C) Velocity and wall thickness of uninjured right carotid artery ( $=10$ /group, One-way ANOVA, Tukey's multiple comparison test, Values \pm SD). (D) Representative M-Mode images of uninjured right carotid artery. Brackets show carotid artery size, and arrows point out the measured wall thickness.

Statistical analysis. Data are presented as mean \pm SD. Statistical analysis were performed with Prism 6.1 software (GraphPad). For analyses between more than 2 groups we used 1-way ANOVA followed by Tukey's multiple comparison test. $P$ values of $<0.05$ were considered significant.

\section{Results}

X203 treatment for 2 weeks did not affect blood lipids. To investigate the effect of IL-11 inhibition on neointimal hyperplasia, we performed wire injury in mice randomly assigned to receive either control IgG, the anti-IL-11 antibody X203, or no treatment (Fig. 1A). Since circulating lipids play an important role in arteriosclerosis and neointimal hyperplasia, we first assessed plasma lipids. With short term treatment, there were no differences in triglyceride, total cholesterol, LDL cholesterol or HDL cholesterol levels in mice across experimental groups (Fig. 1B). Furthermore, liver transaminases were similar in all treatment groups (Fig. 1B) suggesting that systemic anti-IL-11 antibody administration did not affect plasma lipids or liver function over the experimental time course.

X203 treatment reduced post-wire injury neointimal hyperplasia. To determine the effect of IL-11 inhibition on neointimal hyperplasia, we performed ultrasound analyses of injured carotid arteries. Wall thickness was significantly reduced in the X203 treated group compared to controls, whilst there were no differences in blood flow velocity (Fig. 2A,B). Interestingly, the ultrasound measurements of right-side, uninjured carotid artery show significant thinning after X203 treatment, while velocity showed no differences (Fig. 2C,D), demonstrating the effect of the X203 treatment on arterial remodeling. Original acquired ultrasound images are now presented in a supplementary figure (Suppl. Fig. 1).

In addition, injury-related plaque (Fig. 3A), neointimal areas (Fig. 3B) were significantly reduced in X203treated mice compared to controls. There were no differences in tunica media area between the treatment groups (Fig. 3C) Representative images are shown in Fig. 3D. Analyzing the right, uninjured carotid arteries, we found no differences in total vessel area (Fig. 3E), intima (Fig. 3F) or media (Fig. 3G). Representative images are shown in Fig. 3H. One out of 12 control carotid arteries (Suppl. Fig. 2A) and one out of 13 IgG-treated carotid arteries developed native atherosclerotic plaques, whereas none of the 14 X203-treated carotid arteries developed native atherosclerotic plaque.

X203 treatment had no effect on post-endothelial injury macrophage injury-related plaque infiltration. Inflammation and macrophages play an important role in arteriosclerosis and vascular restenosis $^{20}$. Therefore, we assessed the effect of IL-11 inhibition on the number of macrophages infiltrating the 
A

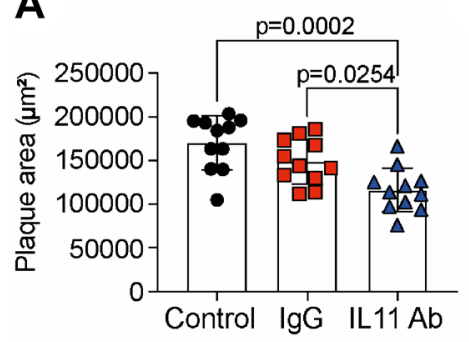

B

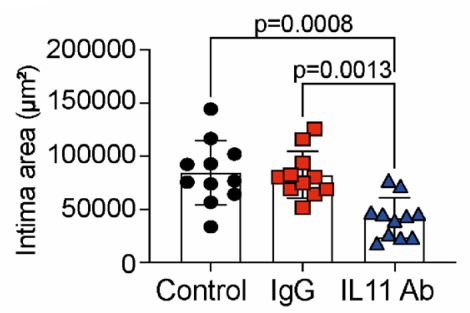

C

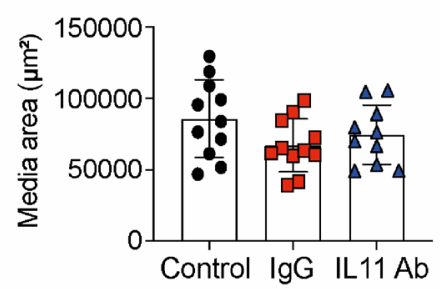

D

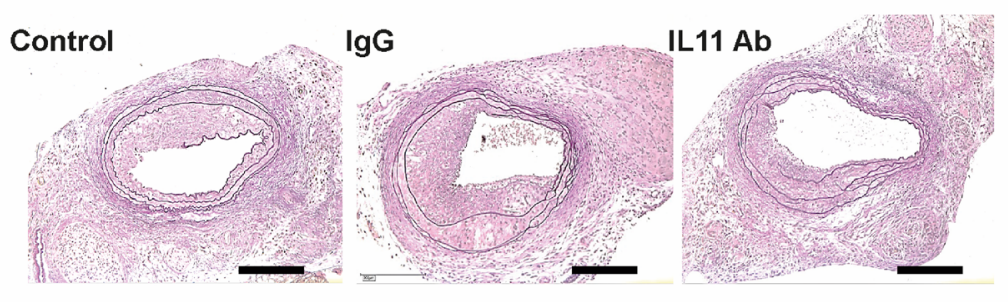

E

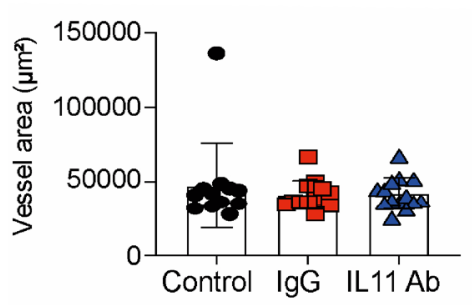

H

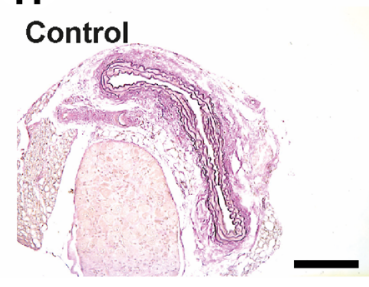

F

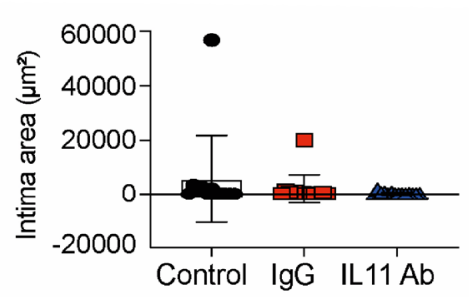

G

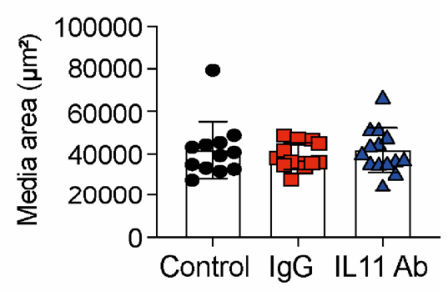

Figure 3. The effect of anti-IL-11 antibody (X203) treatment on neointimal hyperplasia. (A) Injury-related plaque area $(\mathrm{N}=11$ /group, One-way ANOVA, Tukey's multiple comparison test, Values $\pm \mathrm{SD})$. (B) Intima area $(\mathrm{N}=11$ /group, One-way ANOVA, Tukey's multiple comparison test, Values $\pm \mathrm{SD})$. (C) Media area $(\mathrm{N}=11$ /group, One-way ANOVA, Tukey's multiple comparison test, Values \pm SD). (D) Representative images of Verhoeff Van Gieson Elastic stain (scale bar $200 \mu \mathrm{m})$. (E) Vessel area of control uninjured right carotid arteries $(\mathrm{N}=12-14$ / group, One-way ANOVA, Tukey's multiple comparison test, Values \pm SD). (F) Intima area $(\mathrm{N}=12-14$ /group, One-way ANOVA, Tukey's multiple comparison test, Values $\pm \mathrm{SD})$. (G) Media area $(\mathrm{N}=12-14$ /group, One-way ANOVA, Tukey's multiple comparison test, Values \pm SD). (H) Representative images of Verhoeff Van Gieson Elastic stain (scale bar $200 \mu \mathrm{m}$ ).

injury-related plaque area. Neither the proportion of macrophages nor the absolute number of macrophages were different across the treatment groups (Fig. 4A-C). The right carotid arteries showed no staining or isolated subendothelial staining for macrophage marker Mac2 (Fig. 4D), except the one carotid artery from the control groups presenting with native atherosclerotic plaque, which showed predominately macrophages infiltration (Suppl. Fig. 2B).

X203 treatment reduced post-endothelial injury VSMC accumulation. Given the central role of VSMC switching to a synthetic phenotype characterized by proliferation and migration in post-endothelial injury neointimal hyperplasia, we investigated the effect of IL-11 inhibition on VSMC accumulation and phenotype. X203 treatment reduced the numbers of VSMCs in the injury-related plaque (Fig. 5A,B,D), and increased the numbers of VSMCs expressing SM22a, a contractile marker (Fig. 5C,E), when compared to controls, sug- 
A

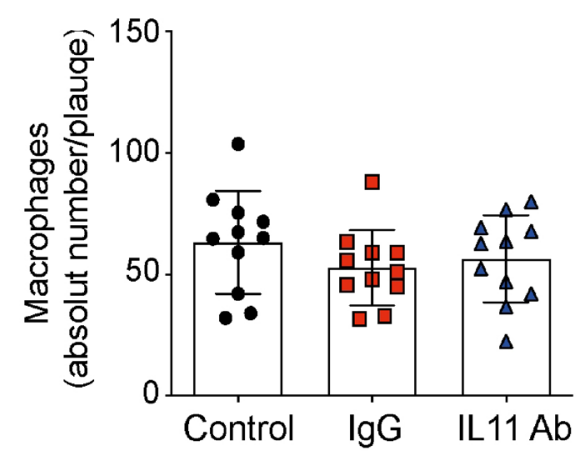

B

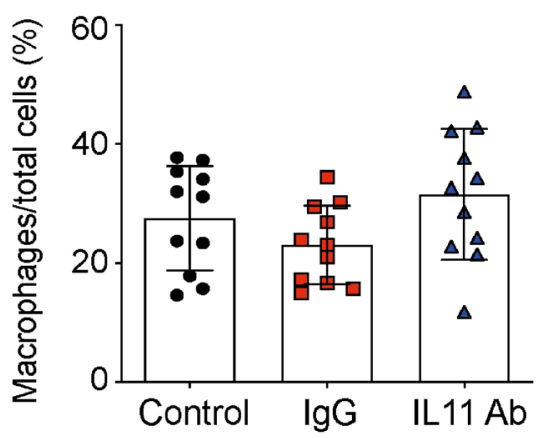

C
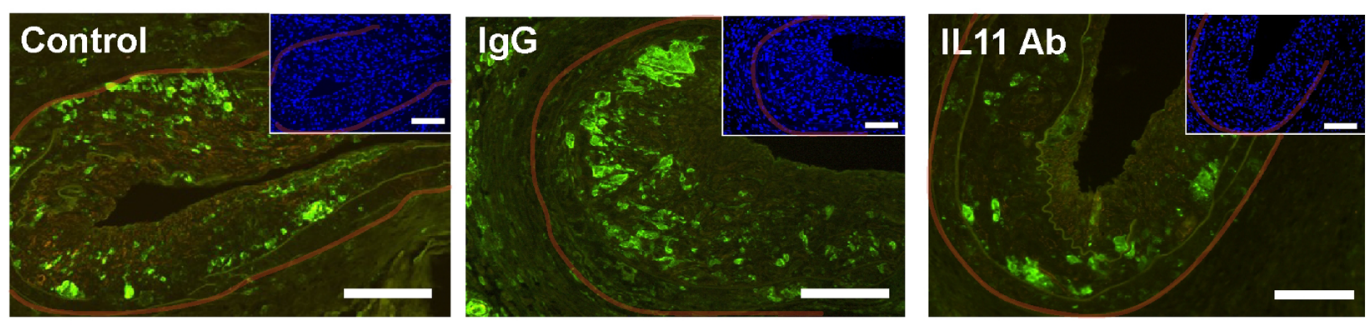

D
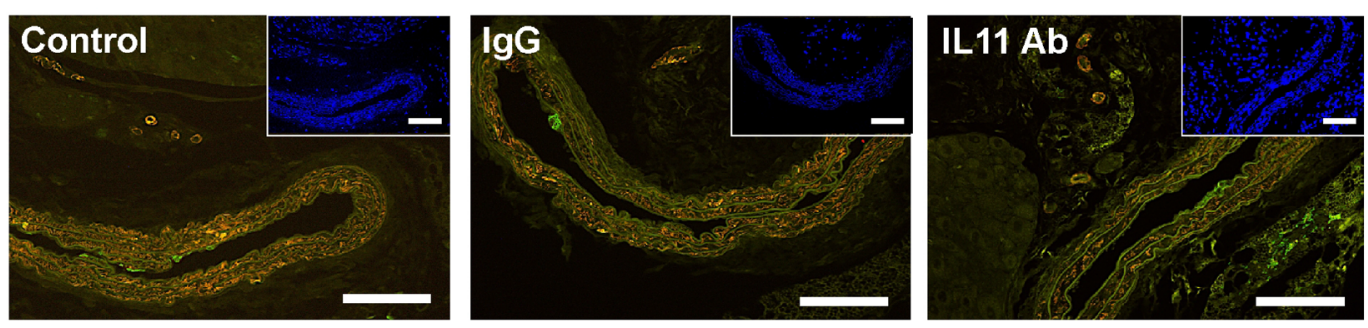

Figure 4. The effect of anti-IL-11 antibody (X203) treatment on macrophage infiltration. (A) Macrophages per injury-related plaque ( $\mathrm{N}=11$ /group, One-way ANOVA, Tukey's multiple comparison test, Values $\pm \mathrm{SD})$. (B) Macrophage proportion of all cells ( $\mathrm{N}=11$ /group, One-way ANOVA, Tukey's multiple comparison test, Values \pm SD). (C) Representative images of Mac2 immunofluorescence staining and corresponding DAPI staining (insets) (scale bar $100 \mu \mathrm{m}$ ). Transparent red-lines were traced to delineate the injury-related plaque area used for quantifications. (D) Representative images of Mac2 staining in the right uninjured carotid arteries and corresponding DAPI staining (insets) (scale bar $100 \mu \mathrm{m})$.

gesting that most VSMCs in the injury-related plaques of the X203-treated mice were contractile in phenotype, suggesting atheroprotection. The right uninjured carotid arteries showed mainly vascular smooth muscle cells in the vessel wall (Fig. 5F).

X203 treatment reduced post-endothelial injury injury-related plaque fibrosis. Several recent studies have reported the pro-fibrotic properties of IL-1 $1^{15,16}$. Given the critical role of VSMC switching to a synthetic phenotype in post-endothelial injury neointimal hyperplasia, which is characterized by the secretion of extracellular matrix, we investigated the effect of IL-11 inhibition on MMP2 expression and collagen content. X203 treatment decreased MMP2 expression (Fig. 6A,B) and reduced collagen content (Fig. 6C,D) in the injuryrelated plaque, compared to controls, again suggesting that inhibition of IL-11 is atheroprotective via reducing VSMC phenotype switching. Interestingly, the uninjured right carotid arteries showed a significant reduction of collagen content after X203 treatment (Fig. 6E,F), demonstrating the anti-fibrotic role of X203 treatment and protection against arterial remodeling. This may account for the right carotid arteries appearing thinner during the ultrasound measurements. 
A

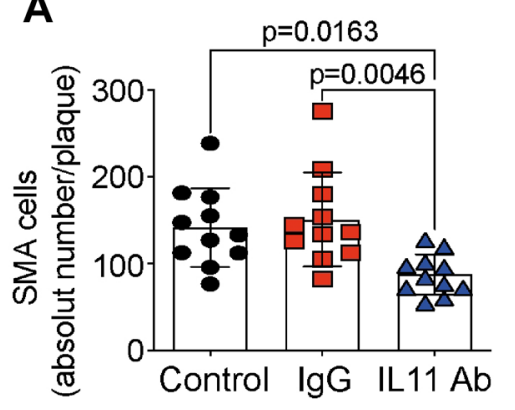

B

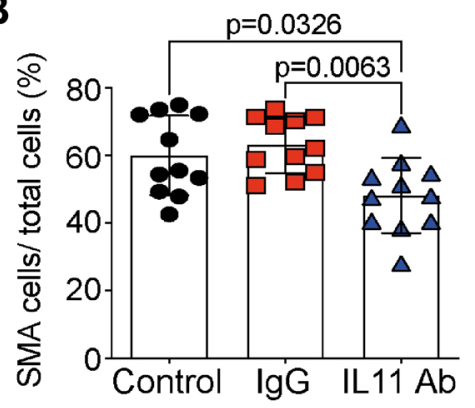

C

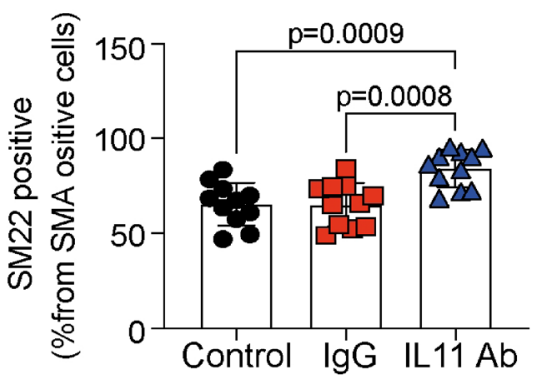

D
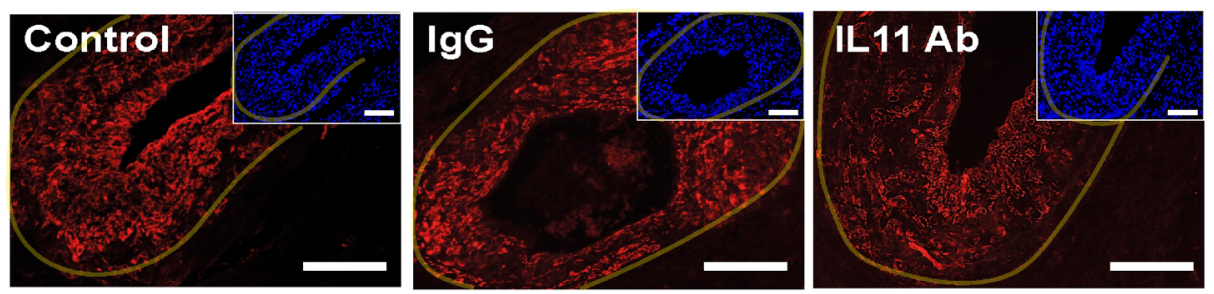

E
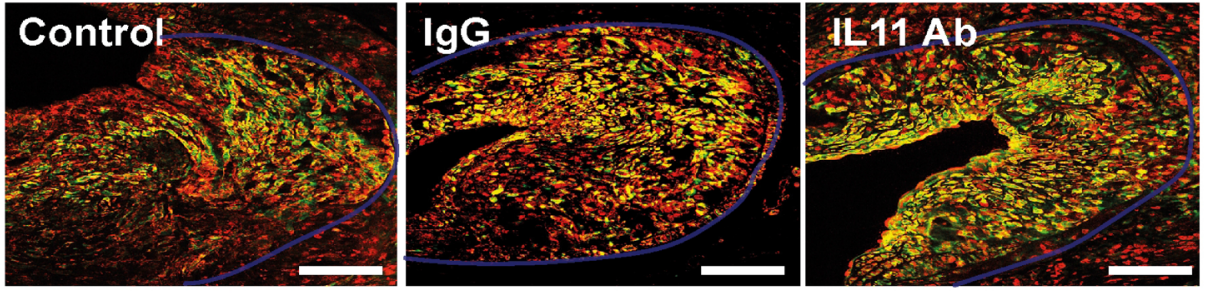

$\mathbf{F}$
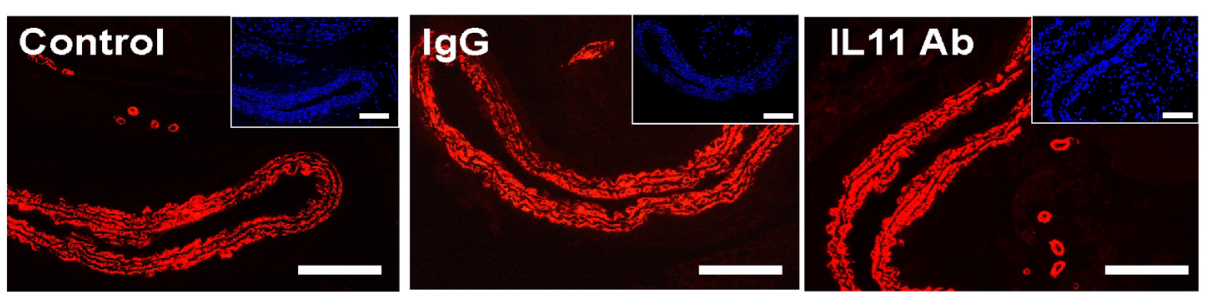

Figure 5. The effect of anti-IL-11 antibody (X203) treatment on injury-related plaque VSMC accumulation. (A) SMA ${ }^{+}$VSMCs per injury-related plaque ( $\mathrm{N}=11$ /group, One-way ANOVA, Tukey's multiple comparison test, Values $\pm \mathrm{SD}$ ). (B) $\mathrm{SMA}^{+}$VSMCs percent of all cells $(\mathrm{N}=11$ /group, One-way ANOVA, Tukey's multiple comparison test, Values $\pm \mathrm{SD}$ ). (C) SM22a expression in VSMCs in the injury-related plaque ( $\mathrm{N}=11$ /group, One-way ANOVA, Tukey's multiple comparison test, Values \pm SD). (D) Representative images of SMA immunofluorescence staining (red) and corresponding DAPI staining (blue, insets) in left, injured carotid arteries (scale bar $100 \mu \mathrm{m}$ ). Transparent yellow-lines were traced to delineate the injury-related plaque area used for quantifications. (E) Representative images of SM22a (green) and SMA (red) immunofluorescence co-staining (yellow, scale bar $50 \mu \mathrm{m}$ ). Transparent blue-lines were traced to delineate the injury-related plaque area used for quantifications. (F) Representative images of SMA immunofluorescence staining (red) and corresponding DAPI staining (blue, insets) in right, uninjured carotid arteries (scale bar $100 \mu \mathrm{m}$ ).

\section{Discussion}

We demonstrate for the first time that inhibition of IL-11 reduces vessel wall thickness and neointimal hyperplasia in a carotid wire-induced endothelial injury mouse model. These findings were associated with beneficial effects on post-endothelial injury injury-related plaque remodelling as evidenced by decreased accumulation of VSMCs, increased proportion of contractile VSMCs, lower MMP2 expression, and reduced collagen content in the injury-related plaque. Interestingly, inhibition of IL-11 reduced the collagen content of uninjured carotid arteries as compared to either IgG or buffer control mice, showing an additional effect on hyperlipidemia-induced arterial remodeling in the absence of mechanical injury. 
A

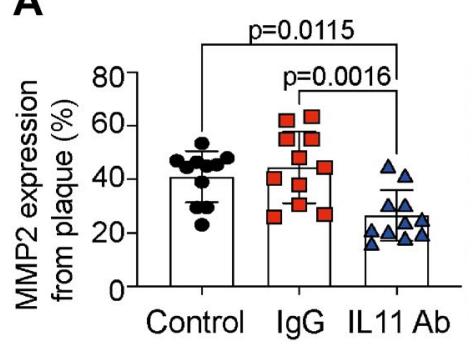

B

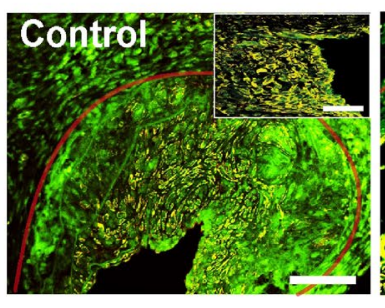

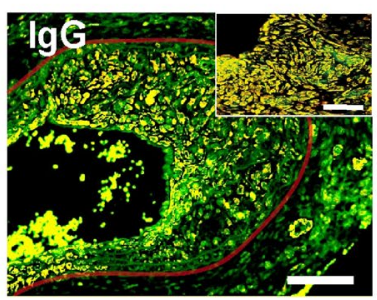

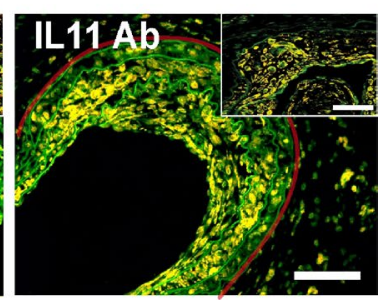

C

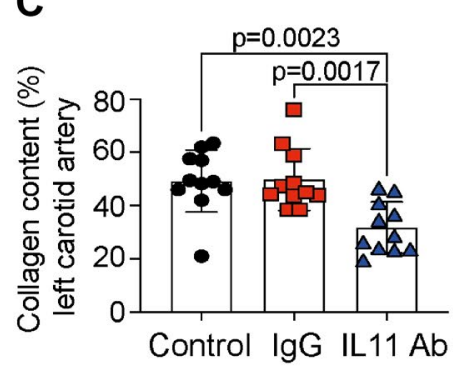

D

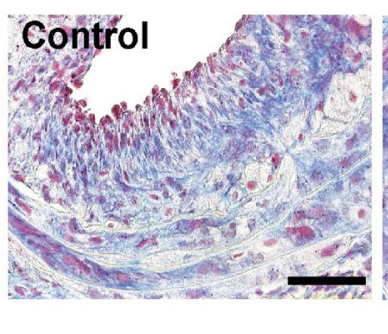

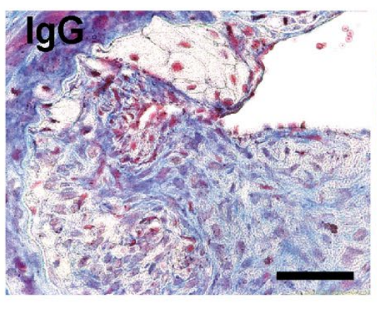

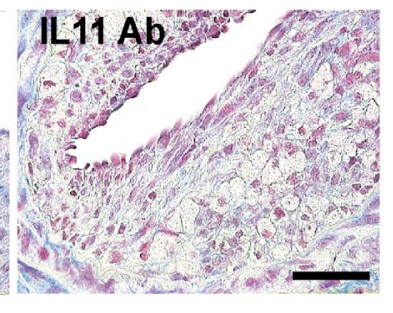

E

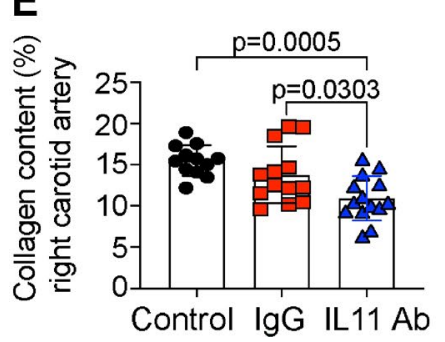

$\mathbf{F}$
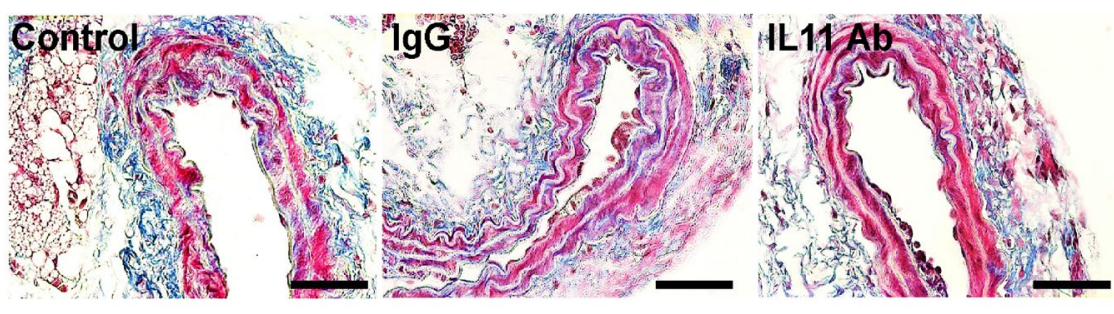

Figure 6. The effect of anti-IL-11 antibody (X203) treatment on post-endothelial injury injury-related plaque fibrosis. (A) MMP2 positive staining per injury-related plaque ( $\mathrm{N}=11$ /group, One-way ANOVA, Tukey's multiple comparison test, Values \pm SD). (B) Representative images of MMP2 immunofluorescence staining (green, scale bar $100 \mu \mathrm{m}$ ). Insets represent higher magnification of positive cells inside the restenosis plaque (scale bar $50 \mu \mathrm{m}$ ). Transparent red-lines were traced to delineate the injury-related plaque area used for quantifications. (C) Collagen content (blue) of the injury-related plaque ( $\mathrm{N}=11$ /group, One-way ANOVA, Tukey's multiple comparison test Values \pm SD). (D) Representative images of Gomori stain (collagen in blue, muscle in red, scale bar $50 \mu \mathrm{m})$. (E) Collagen content (blue) of right, uninjured carotid arteries $(\mathrm{N}=12-14$ / group, One-way ANOVA, Tukey's multiple comparison test Values \pm SD). (F) Representative images of Gomori stain (collagen in blue, muscle in red, scale bar $50 \mu \mathrm{m}$ ).

Autocrine IL-11 signaling is a key downstream effector of TGF $\beta 1$ and ANGII in different cell types involved in extracellular matrix (ECM) production ${ }^{15,16,18,19}$. In cardiac fibroblasts ${ }^{15}$, lung fibroblasts ${ }^{18}$ and hepatic stellate cells ${ }^{19}$, IL-11 is required for ERK-dependent myofibroblast activation. Interestingly, TGF $\beta 1$ induces IL- 11 secretion from aortic and coronary artery VSMCs ${ }^{21,22}$. Recently, we showed a role of IL-11 in phenotypic switching of VSMCs and discovered the existence of an autocrine loop of IL-11 activity in VSMCs, which is required downstream of both TGF $\beta 1$ and ANGII for phenotypic switching to occur in the context of aortic modelling ${ }^{16}$. However, the role of IL-11 in phenotypic switching and function of VSMCs in the setting of neointimal hyperplasia following endothelial injury has not been investigated. It is known that X203 treatment has a positive effect on arterial remodeling in the context of hypertension ${ }^{16}$, on cardiac fibrosis and healing after myocardial infarction $^{15}$, on liver fibrosis ${ }^{19}$ and in idiopathic pulmonary fibrosis ${ }^{18}$.

In this study, we showed that inhibiting IL-11 reduced neointimal proliferation and had favorable effects on injury-related plaque remodelling following wire-induced endothelial injury in the mouse carotid artery. As expected, carotid wire injury induced neointimal hyperplasia in control mice as evidenced by an increase in vessel wall thickness and tunica intima area, and treatment with IgG had no effect on these parameters. Treatment with the anti-IL-11 antibody X203 significantly reduced vessel wall thickness (with no effects on carotid artery velocity) and decreased total injury-related plaque area (with a reduction in tunica intima area but no effect on media area). The reduction in injury-related plaque area with X203 treatment was associated with decreased numbers of VSMCs, with an increased expression of SM22 $\alpha$, a marker for VSMCs with a preserved contractile phenotype ${ }^{23}$. These findings are consistent with our prior study showing that genetic or antibody-mediated inhibition of IL-11 attenuated VSMC phenotypic switching ${ }^{16}$. Several studies have identified macrophages to be important contributors to vascular restenosis ${ }^{24,25}$. However, we found no differences in macrophage injury-related plaque 
infiltration with X203 treatment, suggesting that the beneficial effects of inhibiting IL-11 on reducing neointimal hyperplasia were independent of macrophage accumulation into the injury-related plaque at this timepoint.

In our previous study we showed that IL-11 induces phenotypic switching of VSMCs to a synthetic phenotype characterized by secretion of collagen and extracellular matrix proteins, including MMP2 ${ }^{16}$, which is involved with phenotype switching and migration ${ }^{26,27}$. Furthermore, expression of the VSMC contractile marker SMA22a was also decreased in response to IL-11 antibody treatment in the same study ${ }^{16}$. Consistent with a pathological role for IL-11 on VSMC function, we show here that treatment with X203 decreased injury-related plaque MMP2 levels and increased injury-related plaque SMA22 $\alpha$ levels, suggesting a favorable effect of IL-11 inhibition on vascular remodelling following wire-induced endothelial injury.

We highlight that while IL-11 was discovered three decades ago, there is very little known of its effects in the vasculature. Limited earlier studies have suggested IL-11 as anti-inflammatory, anti-fibrotic and pro-regenerative $^{28}$ and in the vasculature, IL-11 has been thought to inhibit VSMC proliferation and plaque formation ${ }^{29}$, the opposite of what we demonstrate here. One reason for the general misunderstanding of IL-11 function relates to the repeated use of recombinant human IL-11 in mouse models of disease. Paradoxically, it was recently shown that rhIL-11 is a competitive inhibitor of mouse IL-11 in mouse cells and thus much of the earlier literature may need to be reconsidered ${ }^{30}$.

In conclusion, we show for the first time that inhibition of IL-11 reduced neointimal hyperplasia following endothelial injury and had favorable effects on vascular remodelling. These findings position the IL-11 antibody (X203) as a novel therapeutic strategy for preventing post-angioplasty/stent restenosis and improving outcomes in CAD and PAD patients undergoing revascularization.

Received: 11 May 2021; Accepted: 24 September 2021

Published online: 19 October 2021

\section{References}

1. Lee, M. S. \& Banka, G. In-stent restenosis. Interv. Cardiol. Clin. 5, 211-220 (2016).

2. Osadnik, T. et al. The relationships between polymorphisms in genes encoding the growth factors TGF-betal, PDGFB, EGF, bFGF and VEGF-A and the restenosis process in patients with stable coronary artery disease treated with bare metal stent. PLoS ONE 11, e0150500 (2016).

3. Pallero, M. A. et al. Stainless steel ions stimulate increased thrombospondin-1-dependent TGF-beta activation by vascular smooth muscle cells: implications for in-stent restenosis. J. Vasc. Res. 47, 309-322 (2010).

4. Nicolais, C. et al. Therapeutic Options for In-Stent Restenosis. Curr. Cardiol. Rep. 20, 7 (2018).

5. van Varik, B. J. et al. Mechanisms of arterial remodeling: Lessons from genetic diseases. Front. Genet. 3, 290 (2012).

6. Alexander, M. R. \& Owens, G. K. Epigenetic control of smooth muscle cell differentiation and phenotypic switching in vascular development and disease. Annu. Rev. Physiol. 74, 13-40 (2012).

7. Kuang, S. Q. et al. Aortic remodeling after transverse aortic constriction in mice is attenuated with AT1 receptor blockade. Arterioscler. Thromb. Vasc. Biol. 33, 2172-2179 (2013).

8. Elia, L. et al. UHRF1 epigenetically orchestrates smooth muscle cell plasticity in arterial disease. J. Clin. Invest. 128, 2473-2486 (2018).

9. Ren, X. S. et al. NLRP3 gene deletion attenuates angiotensin II-induced phenotypic transformation of vascular smooth muscle cells and vascular remodeling. Cell Physiol. Biochem. 44, 2269-2280 (2017).

10. Zhang, Z. et al. Upregulation of TRPM7 channels by angiotensin II triggers phenotypic switching of vascular smooth muscle cells of ascending aorta. Circ. Res. 111, 1137-1146 (2012).

11. Clement, M. et al. Vascular smooth muscle cell plasticity and autophagy in dissecting aortic aneurysms. Arterioscler. Thromb. Vasc. Biol. 39, 1149-1159 (2019).

12. Gallo, E. M. et al. Angiotensin II-dependent TGF-beta signaling contributes to Loeys-Dietz syndrome vascular pathogenesis. J. Clin. Invest. 124, 448-460 (2014).

13. Holm, T. M. et al. Noncanonical TGFbeta signaling contributes to aortic aneurysm progression in Marfan syndrome mice. Science 332, 358-361 (2011)

14. Ford, C. M., Li, S. \& Pickering, J. G. Angiotensin II stimulates collagen synthesis in human vascular smooth muscle cells. Involvement of the AT(1) receptor, transforming growth factor-beta, and tyrosine phosphorylation. Arterioscler. Thromb. Vasc. Biol. 19, 1843-1851 (1999).

15. Schafer, S. et al. IL-11 is a crucial determinant of cardiovascular fibrosis. Nature 552, 110-115 (2017).

16. Lim, W. W. et al. Interleukin-11 is important for vascular smooth muscle phenotypic switching and aortic inflammation, fibrosis and remodeling in mouse models. Sci. Rep. 10, 17853 (2020).

17. Curaj, A., Zhoujun, W., Staudt, M. \& Liehn, E. A. Induction of accelerated atherosclerosis in mice: The "wire-injury" model. J. Vis. Exp. 162, e54571 (2020).

18. Ng, B. et al. Interleukin-11 is a therapeutic target in idiopathic pulmonary fibrosis. Sci. Transl. Med. 11, eaaw1237 (2019).

19. Widjaja, A. A. et al. Inhibiting interleukin 11 signaling reduces hepatocyte death and liver fibrosis, inflammation, and steatosis in mouse models of nonalcoholic steatohepatitis. Gastroenterology 157, 777-792 (2019).

20. Frodermann, V. \& Nahrendorf, M. Macrophages and cardiovascular health. Physiol. Rev. 98, 2523-2569 (2018).

21. Lebastchi, A. H. et al. Activation of human vascular cells decreases their expression of transforming growth factor-beta. Atherosclerosis 219, 417-424 (2011).

22. Taki, H. et al. Monokine stimulation of interleukin-11 production by human vascular smooth muscle cells in vitro. Atherosclerosis 144, 375-380 (1999).

23. Halayko, A. J. et al. Divergent differentiation paths in airway smooth muscle culture: Induction of functionally contractile myocytes. Am. J. Physiol. 276, 197-206 (1999).

24. Zhang, M. et al. In-stent restenosis is associated with neointimal angiogenesis and macrophage infiltrates. Pathol. Res. Pract. 210, 1026-1030 (2014).

25. Yahagi, K. et al. Pathophysiology of native coronary, vein graft, and in-stent atherosclerosis. Nat. Rev. Cardiol. 13, 79-98 (2016).

26. Vigetti, D. et al. Matrix metalloproteinase 2 and tissue inhibitors of metalloproteinases regulate human aortic smooth muscle cell migration during in vitro aging. FASEB 20, 1118-1130 (2006).

27. Risinger, G. M., Hunt, T. S., Updike, D. L., Bullen, E. C. \& Howard, E. W. Matrix metalloproteinase-2 expression by vascular smooth muscle cells is mediated by both stimulatory and inhibitory signals in response to growth factors. J. Biol. Chem. 281, 25915-25925 (2006). 
28. Cook, S. A. \& Sebastian, S. S. Hiding in plain sight: Interleukin-11 emerges as a master regulator of fibrosis, tissue integrity, and stromal inflammation. Annu. Rev. Med. 27(71), 263-276 (2020).

29. Zimmerman, M. A. et al. Interleukin-11 attenuates human vascular smooth muscle cell proliferation. Am. J. Physiol. Heart Circ. Physiol. 283(1), H175-H180 (2002).

30. Widjaja, A. A. et al. Redefining IL11 as a regeneration-limiting hepatotoxin and therapeutic target in acetaminophen-induced liver injury. Sci. Transl. Med. 13(597), eaba8146 (2021).

\section{Acknowledgements}

We thank Roya Soltan and Melanie Garbe for excellent technical assistance.

\section{Author contributions}

Conceptualization: D.J.H., S.A.C.; Methodology: E.A.L., D.S., R.R.S., S.A.C., D.J.H.; Formal analysis and investigation: D.S., E.A.L., P.N., D.C.M., C.R., K.A., G.C., S.H.R., R.R.S.; Writing-original draft preparation: D.S., E.A.L.; Writing—review and editing: R.R.S., D.J.H., S.A.C.; Funding acquisition: D.J.H.; Resources: D.J.H., E.A.L., S.A.C.; Supervision: D.J.H., S.A.C. All authors commented on previous versions of the manuscript and all authors read and approved the final manuscript.

\section{Funding}

This study was supported by the Interdisciplinary Centre for Clinical Research IZKF Aachen (junior research group to E.A.L.). DS is supported by the Clinician Scientist program of the Faculty of Medicine of the RWTH Aachen University. RRS was supported by the Agency for Science, Research and Technology (A*STAR) and the National University of Singapore (NUS). SHR is supported by the Singapore Ministry of Health's National Medical Research Council under its Open Fund-Young Individual Research Grant (OF-YIRG)-(NMRC/ OFYIRG/0078/2018). SAC is supported by NMRC STaR award and the Tanoto Foundation. DJH is supported by the Duke-NUS Signature Research Programme funded by the Ministry of Health, Singapore Ministry of Health's National Medical Research Council under its Clinician Scientist-Senior Investigator scheme (NMRC/ CSA-SI/0011/2017), Centre Grant (CGAug16M006), and Collaborative Centre Grant scheme (NMRC/ CGAug16C006). This article is based upon work from COST Action EU-CARDIOPROTECTION CA16225 supported by COST (European Cooperation in Science and Technology).

\section{Competing interests}

SAC is an inventor on the patent applications: WO/2017/103108 (TREATMENT OF FIBROSIS), WO/2018/109174 (IL-11 ANTIBODIES), WO/2018/109170 (IL-11RA ANTIBODIES). S.A.C. is a shareholder of Enleofen Bio PTE LTD. The remaining authors (DS, EAL PN, DCM, CR, KA, GEC, SHR, RRS and DJH) declare no competing interests.

\section{Additional information}

Supplementary Information The online version contains supplementary material available at https://doi.org/ 10.1038/s41598-021-99880-y.

Correspondence and requests for materials should be addressed to D.J.H.

Reprints and permissions information is available at www.nature.com/reprints.

Publisher's note Springer Nature remains neutral with regard to jurisdictional claims in published maps and institutional affiliations.

Open Access This article is licensed under a Creative Commons Attribution 4.0 International License, which permits use, sharing, adaptation, distribution and reproduction in any medium or format, as long as you give appropriate credit to the original author(s) and the source, provide a link to the Creative Commons licence, and indicate if changes were made. The images or other third party material in this article are included in the article's Creative Commons licence, unless indicated otherwise in a credit line to the material. If material is not included in the article's Creative Commons licence and your intended use is not permitted by statutory regulation or exceeds the permitted use, you will need to obtain permission directly from the copyright holder. To view a copy of this licence, visit http://creativecommons.org/licenses/by/4.0/.

(C) The Author(s) 2021 